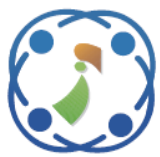

\title{
RSLBO: Random Selected Leader Based Optimizer
}

\author{
Fatemeh Ahmadi Zeidabadi ${ }^{1}$ \\ Mohammad Dehghani ${ }^{2 *}$ \\ Om Parkash Malik ${ }^{3}$ \\ ${ }^{1}$ Department of Mathematics and Computer Sciences, Sirjan University of Technology, Sirjan, Iran \\ ${ }^{2}$ Graduate of the Department of Electrical and Electronics Engineering, \\ Shiraz University of Technology, Shiraz, Iran \\ ${ }^{3}$ Department of Electrical Engineering, University of Calgary, Calgary Alberta, Canada \\ * Corresponding author's Email: adanbax @ gmail.com
}

\begin{abstract}
Designed optimization problems in different disciplines of science should be solved using appropriate techniques. Optimization algorithms are among the most effective and widely used methods in solving optimization problems that are able to provide suitable solutions for these problems. Innovation and scientific contribution of this article in designing a new optimizer called Random Selected Leader Based Optimizer (RSLBO) in order to be used in optimizing the objective functions of optimization problems and achieving the desired solutions. The main idea of the proposed RSLBO is to increase the search power using a random leader. In fact, instead of the algorithm population update relying on several specific members, such as the best member or the worst member, any ordinary member of the population can be a leader in guiding and updating the algorithm population. RSLBO is described, then mathematically modelled to be used in solving optimization problems. The main feature and advantage of the proposed RSLBO is that its implementation and relationships are very simple and understandable, and it also lacks control parameters. The performance of the proposed RSLBO is evaluated on a set of twenty-three standard functions. Also, in order to analyse the quality of the proposed RSLBO in providing appropriate solutions to optimization problems, the results obtained from the proposed algorithm are compared with eight well-known algorithms including Flow Direction Algorithm (FDA), Hide Object Game Optimizer (HOGO), Whale Optimization Algorithm (WOA), Grey Wolf Optimization (GWO), Teaching-Learning-Based Optimization (TLBO), Gravitational Search Algorithm (GSA), Particle Swarm Optimization (PSO), and Genetic Algorithm (GA). The results of optimization of objective functions indicate the high ability of the RSLBO to solve optimization problems and provide appropriate and acceptable solutions. Also, the analysis and comparison of the performance of the eight well-known optimization algorithms against the proposed RSLBO shows that the RSLBO has provided more appropriate solutions and is superior and much more competitive than the eight compared algorithms.
\end{abstract}

Keywords: Optimization, Optimization problem, Population-based, Random, Random leader.

\section{Introduction}

Optimization means finding the most appropriate solution to a problem that has several possible solutions. An optimization problem is modeled using the three main parts of variables, objective functions, and constraints [1]. After mathematical modeling for an optimization problem, the optimal solution for that problem must be obtained using the appropriate technique. Optimization problem solving methods include two groups of deterministic methods and stochastic methods [2]. deterministic methods use derivative and gradients information to find the optimal solution [3]. The advantage of these methods is that it ensures that the solution obtained is the best solution to the problem. But the inability to solve: complex problems, nonlinear functions, highdimensional search spaces, and time consuming are the main disadvantages of these methods. In contrast to deterministic methods, stochastic methods are able to provide appropriate solutions to optimization problems without the need for derivative and gradient information and only based on random scan of the search space [4]. Optimization algorithms are one of 
the most widely used stochastic methods in solving optimization problems that provide acceptable and reasonable solutions to optimization problems in a repetition-based process [5]. The performance of most optimization algorithms is such that they first suggest a number of random and feasible solutions to the optimization problem. Then, in an iterative process based on the steps of the algorithm, they improve the proposed solutions. At the end of the iterations, the optimization algorithm proposes the best solution it has been able to obtain as the solution to the optimization problem [6]. Every optimization problem has a main way called global optimal. The important thing about optimization algorithms is that the solutions obtained from these methods are not necessarily global optimal. For this reason, the solutions obtained from optimization algorithms are called quasi-optimal solutions [7]. Therefore, in comparison of the performance of several algorithms on optimizing a problem, a superior algorithm is that algorithm which provides a more appropriate quasioptimal solution. This has led to the introduction of various optimization algorithms by researchers in order to provide quasi-optimal solutions. In addition, optimization algorithms have been applied in various fields in the literature such as energy [8-11], protection [12], electrical engineering [13-18], and energy carriers $[19,20]$ to achieve the optimal solution.

Innovation and scientific contribution of this paper in the design of a new optimizer called Random Selected Leader Based Optimizer (RSLBO). The main idea in designing the proposed RSLBO is to prevent the algorithm from relying on specific members such as the best member of the population and to more effectively update members of the population based on random leaders. The advantage of this method is increasing the search power in the problem-solving space and achieving quasi-optimal solutions. The various stages of the proposed RSLBO are described and its mathematical model is presented. The performance of the RSLBO in solving optimization problems is implemented on a standard set consisting of twenty-three standard functions. The results of the proposed algorithm are also compared with eight well-known algorithms.

The rest of the article is organized in such a way that in Section 2, an overview of optimization algorithms is presented. In section 3, the proposed algorithm is introduced. Simulation studies are presented in Section 4. Conclusions and several suggestions for future studies are provided in Section 5.

\section{Background}

Optimization algorithms are among the stochastic methods of solving optimization problems that are able to provide suitable solutions to these problems. optimization algorithms based on the main idea in their design can be divided into four groups: swarmbased, physics-based, game-based, and evolutionarybased optimization algorithms.

Swarm-based optimization algorithms are developed based on simulations of the natural behaviors of animals, plants, insects, and other living things in nature. Particle Swarm Optimization (PSO) is one of the oldest optimization algorithms that belongs to this group. PSO is designed based on modeling the natural behavior of birds and fish [21]. Simulation of interactions between patients and doctor has been used in the design of the Doctor and Patients Optimization (DPO) [22]. Modeling the natural behaviors of gray wolves when hunting, as well as hierarchical leadership of wolves, has been used in the design of the Grey Wolf Optimization (GWO) [23]. Simulation of student and teacher behaviors in the classroom space and educational system has been used to introduce TeachingLearning-Based Optimization (TLBO) [24]. Whale Optimization Algorithm (WOA) is a nature-inspired meta-heuristic optimization algorithm, which mimics the social behavior of humpback whales and is inspired by the bubble-net hunting strategy [25]. Some other algorithms in this group are: Two Stage Algorithm(TSO) [26], Group Mean Based Optimizer (GMBO) [27], Donkey Theorem Optimization (DTO) [28], Good and Bad Groups Based Optimizer (GBGBO) [29], Multi Leader Optimizer (MLO) [2], Good and Bad and Ugly Optimizer (GBUO) [30], and Following Optimization Algorithm (FOA) [31].

Physics-based optimization algorithm are introduced based on modeling different laws of physics and physical processes. Simulated Annealing (SA) is one of the oldest and most famous algorithms in this group, which is designed based on imitation the process of gradual heating and cooling of metals [32]. Gravitational force law simulation between objects at different distances from each other is used in the design of Gravitational Search Algorithm (GSA) [33]. Hook law modeling in a system involving weights that are connected to each other with different springs is used to introduce Spring Search Algorithm (SSA) [7, 34]. Flow Direction Algorithm (FDA), is a physics-based algorithm which mimics the flow direction to the outlet point with the lowest height in a drainage basin. In other words, flow moves to neighbor with lowest high or best objective function [35]. Some other algorithms 
in this group are: Momentum Search Algorithm (MSA) [36], Binary Spring Search Algorithm (BSSA) [37], Henry Gas Solubility Optimization (HGSO) [38], and Central Force Optimization (CFO) [39].

Game-based optimization algorithms are developed based on modeling the rules of different individual and group games and player behavior. Football game league simulations and club behavior are used in the design of the Football Game Based Optimization (FGBO) [40]. Modeling player behavior and game rules in the game finding the hidden object are used in the design of Hide Object Game Optimizer (HOGO) [41]. In designing the Darts Game Optimizer (DGO), the rules of darts and players are imitated in this game [42]. Some other algorithms in this group are: Orientation Search Algorithm (OSA) [43], Ring Game Based Optimizer (RTGBO) [44], Dice Game Optimization (DGO) [14], and Shel Game Optimization (SGO) [45].

Evolutionary-based optimization algorithms are developed based on simulations of genetics and evolutionary laws. Genetic Algorithm (GA) is one of the oldest and most widely used evolutionary-based optimization algorithms. GA is designed based on reproductive process simulation and Darwin's theory of evolutionary, using operators of selection, crossover, and mutation [46]. Artificial Immune System (AIS) has been developed based on simulating the behavior of the immune system and the body's defense against disease[47]. Some other algorithms in this group are: Evolutionary Programming (EP) [48], Artificial Infectious Disease (AID) [49], Evolution Strategy (ES) [50], Genetic Programming (GP) [51], Cultural Algorithm [52], Differential Evolution (DE) [53], and BiogeographyBased Optimizer (BBO) [54].

Optimization algorithms have been developed and improved to provide more suitable quasi-optimal solutions. Although from a theoretical point of view we can point to the differences in the main idea, the complexity of the equations or having control parameters, but the most important indicator in comparing the performance of optimization algorithms is the value of the objective function. In fact, the criterion of superiority of one algorithm over another algorithm in solving an optimization problem is the value of the objective function. Another point about optimization algorithms is that an algorithm may perform very well in solving one problem but failing to solve another. Therefore, several algorithms can be compared when implemented on solving an optimization problem and the results of the objective function values are available.

\section{Random selected leader based optimizer}

In this section, the proposed optimizer is presented. Random Selected Leader Based Optimizer (RSLBO) is a population-based method that is designed to be used in solving various optimization problems. The search space of each optimization problem has dimensions proportional to the number of variables of that problem. Therefore, for each variable of the problem, a coordinate axis is considered. Members of the algorithm population move in the search space as search agents. Based on the position of each member of the population in the search space, the values of the problem variables are specified on the coordinate axes. Thus, each member of the population is a vector that proposes values for the problem variables. The total population of the algorithm can be represented by the juxtaposition of these vectors as a matrix called the population matrix using Eq. (1).

$$
X=\left[\begin{array}{c}
X_{1} \\
\vdots \\
X_{i} \\
\vdots \\
X_{N}
\end{array}\right]_{N \times m}=\left[\begin{array}{ccccc}
x_{1,1} & \cdots & x_{1, d} & \cdots & x_{1, m} \\
\vdots & \ddots & \vdots & \ddots & \vdots \\
x_{i, 1} & \cdots & x_{i, d} & \cdots & x_{i, m} \\
\vdots & \ddots & \vdots & \ddots & \vdots \\
x_{N, 1} & \cdots & x_{N, d} & \cdots & x_{N, m}
\end{array}\right]_{N \times m}
$$

Here, $X$ is the population matrix of RSLBO, $X_{i}$ is the $i$ th member of RSLBO, $N$ is the number of population members, $m$ is the number of problem variables, $x_{i, d}$ is the value for $d^{\prime}$ th variable specified by $i$ 'th member.

As mentioned, each member of the population is a solution to the problem that sets values for the problem variables. Based on the values provided by each member of the population, the objective function is evaluated and a value is calculated for the objective function. Therefore, the objective function can be represented as a vector or using Eq. (2).

$$
F=\left[\begin{array}{c}
f_{1} \\
\vdots \\
f_{i} \\
\vdots \\
f_{N}
\end{array}\right]_{N \times 1}=\left[\begin{array}{c}
F\left(X_{1}\right) \\
\vdots \\
F\left(X_{i}\right) \\
\vdots \\
F\left(X_{N}\right)
\end{array}\right]_{N \times 1}
$$

Here, $F$ is the vector of objective function. The minimum value obtained for the objective function can determine the candidate for the appropriate solution to the optimization problem.

The process of updating the population in the RSLBO is independent of relying on specific members of the population, based on the random choice of the leader to guide each member in the search space. In the proposed algorithm, a member is 
randomly selected to update the position of each member of the population.

The above concepts and the process of population updating are mathematically modeled using Eqs. (3) to (7).

$$
\begin{gathered}
R S L_{i}=X_{k} k \in\{1,2,3, \ldots, N\} \\
d x_{i, d}=\left\{\begin{array}{c}
\left(R S L_{i, d}-I \times x_{i, d}\right), \quad F_{R S L_{i}}<F_{i} \\
\left(x_{i, d}-I \times R S L_{i, d}\right), \quad \text { else }
\end{array}\right. \\
X_{i}^{\text {new }}=X_{i}+r \times d X_{i} \\
X_{i}=\left\{\begin{array}{c}
X_{i}^{\text {new }}, F_{i}^{\text {new }}<F_{i} \\
X_{i}, \text { else }
\end{array}\right. \\
I=\text { round }(1+\text { rand })
\end{gathered}
$$

Here, $R S L_{i}$ is the random selected leader for guiding $i$ 'th member and $F_{R S L_{i}}$ is the its objective function value. $k$ is the row number of the selected member as leader, $d x_{i, d}$ is the value of the displacement of the $i$ 'th member in the search space on $d^{\prime}$ 'th axis, $X_{i}^{\text {new }}$ is the suggestion new status of $i$ 'th member in search space and $F_{i}^{\text {new }}$ is its objective function value. $I$ is a random number which is 1 or 2 , $r$ is a random number in interval $[0-1]$.

The proposed RSLBO is an iteration-based method. The process of updating population members is repeated based on Eqs. (3) to (7) and until the cessation condition is reached. After the algorithm is fully implemented on the considered optimization problem, the RSLBO presents the best quasi-optimal solution obtained. The flowchart of the various stages of the proposed RSLBO is drawn in Fig. 1.

\section{Simulation studies}

In this section, the ability of the proposed RSLBO to optimize and provide suitable quasi-optimal solutions for optimization problems is analysed. For this purpose, a standard set consisting of twenty-three objective functions of different types of unimodal, high-dimensional multimodal, and fixed-dimensional multimodal is considered. In order to analyse the quality and power of the RSLBO, the results are compared with eight well-known algorithms including Flow Direction Algorithm (FDA) [35], Hide Object Game Optimizer (HOGO) [41], Whale Optimization Algorithm (WOA) [25], Grey Wolf

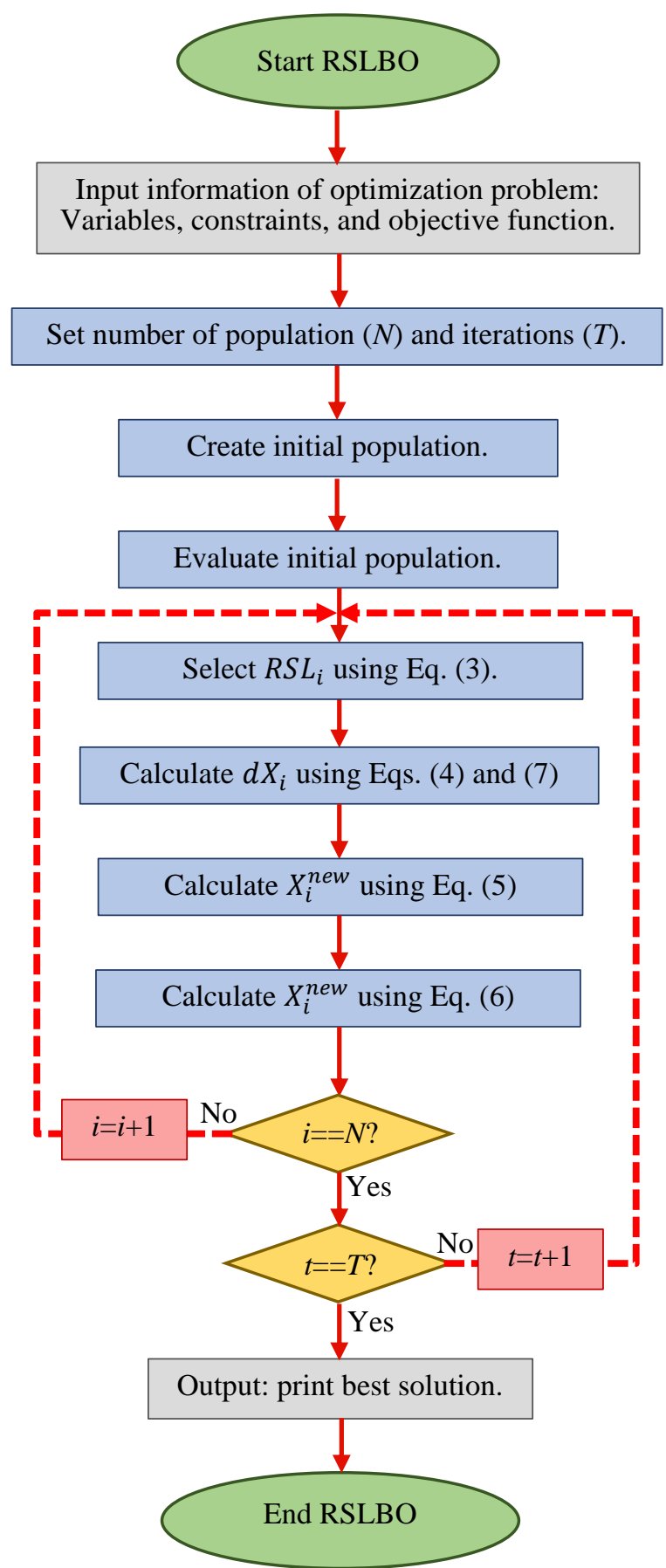

Figure. 1 Flowchart of RSLBO

Optimization (GWO) [23], Teaching-LearningBased Optimization (TLBO) [24], Gravitational Search Algorithm (GSA) [33], Particle Swarm Optimization (PSO) [21], and Genetic Algorithm (GA) [46]. The performance of optimization algorithms in solving these functions has been reported using two indicators of the average of the best solutions (ave) and the standard deviation of these solutions (std).

Seven objective functions $\mathrm{F} 1$ to $\mathrm{F} 7$ of the unimodal type have been selected to analyse the capability of the optimization algorithms. The results 
of optimization of the unimodal objective functions using the RSLBO and eight compared algorithms are presented in Table 1. Based on the results of this table, the proposed RSLBO has a high capability in achieving quasi-optimal solutions. Also, the comparison of the results shows that the RSLBO has a better performance than the eight compared algorithms and is superior to them.

Six F8 to F13 objective functions have been selected to investigate the capability of optimization algorithms in solving high-dimensional multimodal problems. The optimization results of this type of function using the proposed RSLBO and the compared optimization algorithms are presented in Table 2. The results obtained from the RSLBO indicate its acceptable ability to optimize multivariate functions. Comparison of the performance of the optimized algorithms compared to the RSLBO shows that the proposed algorithm has a better performance in optimizing this type of functions.

Ten objective functions of F14 to F23 of fixeddimensional multimodal type have been selected to evaluate the performance of optimization algorithms. The results of optimization of this type of objective functions are presented in Table 3 . The results of this table show that the proposed RSLBO has a very high capability in solving this type of objective functions. Also, the analysis and comparison of the performance of the RSLBO compared to the results obtained from the eight compared algorithms shows that the proposed algorithm is able to provide quasi-optimal solutions more effectively.

\subsection{Discussion}

Two important indicators of exploitation and exploration have a special role in evaluating the performance of optimization algorithms. Exploitation demonstrates the power of algorithms in providing quasi-optimal and near to global solutions. In fact, an optimization algorithm should be able to provide a solution that is as close to the global optimal as possible after it has been fully implemented on an optimization problem. It is clear that the closer the solution obtained from the algorithm is to the global optimal, algorithm has higher exploitation power. The index of exploitation is especially important in problems that have only one main solution. F1 to F7 unimodal functions with only one main solution are suitable for analyzing the exploitation index. Based on the optimization results of these functions presented in Table 1, it is clear that the proposed algorithm has a high power in the exploitation index and has been able to provide solutions very close to the global optimal. Exploration demonstrates the algorithm's ability to accurately and effectively search the search space of optimization problem. This index means that an algorithm must be able to scan various areas of the search space well during algorithm replications to avoid getting stuck in optimal local areas. This index is especially important in optimization problems that have several local solutions in addition to the main solution. Multimodal F8 to F23 functions also have locally optimal solutions and are therefore very suitable for evaluating the exploration index in optimization algorithms. What is clear from the optimization results of these functions in Tables 2 and 3 is that the proposed algorithm has high power in accurately scanning the search space and passing through local solutions. Therefore, the proposed algorithm has much better conditions in the exploration index than the eight compared algorithms.

\section{Conclusions and future studies}

There are countless optimization problems in various disciplines of science that need to be solved using optimization methods. Optimization algorithms are able to offer suitable solutions to optimization problems by randomly scanning the search space. In this paper, a new optimizer called Random Selected Leader Based Optimizer (RSLBO) was introduced to be used in solving optimization problems. The main idea in designing this algorithm was to update the population members based on random leaders in order to guide the population in the search space. In fact, in each iteration of the algorithm, a member is randomly selected as the leader to update the position of a member of the population. The advantage of this process is that the algorithm does not rely on a specific member of the population and does not tend to areas around that particular member. The various stages of the proposed RSLBO were described and its mathematical model was presented for use in solving optimization problems. The ability of the proposed RSLBO to provide suitable solutions for optimization problems was tested on a standard set consisting of twenty-three different objective functions from different types of unimodal, high-dimensional multimodal, and fixed-dimensional multimodal functions. The results of optimization of unimodal functions showed the high exploitation power of the proposed RSLBO in achieving suitable quasi-optimal solutions and close to the global optimal. The results of optimization of multimodal objective functions showed that the RSLBO has a high power in the index of exploration and passing through the optimal areas in the search space. Also, the performance of the RSLBO in optimization was compared with the 
Table 1. Results of RSLBO and other algorithms for unimodal test functions

\begin{tabular}{|c|c|c|c|c|c|c|c|c|c|c|c|c|c|c|}
\hline 7 & & $\sigma^{T}$ & & T & & ${ }^{T}$ & & $T$ & & $T_{T}$ & & 7 & & \\
\hline & 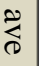 & $\frac{n}{2}$ & $\stackrel{\overbrace{}}{\text { ¿ }}$ & $\frac{n}{\vec{a}}$ & $\stackrel{\varkappa}{\varkappa}$ & $\frac{n}{2}$ & 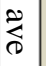 & $\frac{n}{2}$ & 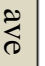 & $\frac{n}{2}$ & 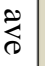 & $\frac{n}{2}$ & 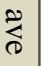 & \\
\hline $\begin{array}{l}v \\
\dot{\vec{t}} \\
\dot{x} \\
\overline{0} \\
\frac{1}{\omega} \\
\end{array}$ & 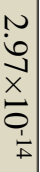 & & 0 & 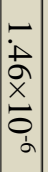 & 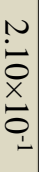 & $\begin{array}{l}-\dot{a} \\
\hat{N} \\
\times \\
0 \\
0 \\
\pm\end{array}$ & $\begin{array}{l}0 \\
\dot{\infty} \\
x \\
\overrightarrow{0} \\
\dot{\omega} \\
\alpha\end{array}$ & 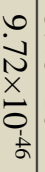 & $\begin{array}{l}\omega \\
0 \\
0 \\
x \\
x \\
0 \\
\frac{1}{N}\end{array}$ & $\begin{array}{l}a \\
\dot{0} \\
\frac{1}{x} \\
\overrightarrow{0} \\
\frac{1}{N}\end{array}$ & $\begin{array}{l}a \\
\dot{\omega} \\
\dot{x} \\
\overrightarrow{0} \\
\vdots \\
\vdots \\
\omega\end{array}$ & $\begin{array}{c}\overrightarrow{\tilde{N}} \\
\vec{x} \\
\underset{0}{0} \\
\substack{\infty \\
\infty}\end{array}$ & $\begin{array}{l}n \\
\hat{\infty} \\
x \\
x \\
0 \\
\frac{1}{4}\end{array}$ & O \\
\hline & $\begin{array}{l}0 \\
8 \\
\infty \\
\infty\end{array}$ & $\begin{array}{l}0 \\
\frac{0}{0} \\
\vec{A}\end{array}$ & $\begin{array}{l}\stackrel{0}{0} \\
\dot{\omega} \\
\infty \\
\infty\end{array}$ & $\mid \begin{array}{l}0 \\
\stackrel{\perp}{N} \\
\stackrel{N}{0}\end{array}$ & 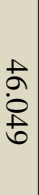 & $\begin{array}{l}0 \\
\dot{u} \\
u \\
x \\
0 \\
0 \\
b\end{array}$ & $\begin{array}{l}N \\
a \\
x \\
0 \\
0 \\
\infty\end{array}$ & $\stackrel{0}{+}$ & $\begin{array}{l}0 \\
0 \\
\infty \\
\propto \\
\not\end{array}$ & $\begin{array}{l}\vec{D} \\
\vec{N} \\
x \\
\dot{0} \\
\overrightarrow{0} \\
\frac{1}{N}\end{array}$ & $\begin{array}{l}\vec{i} \\
\vec{y} \\
x \\
\overrightarrow{0} \\
\frac{1}{v}\end{array}$ & 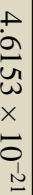 & 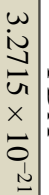 & $\mathbb{T}$ \\
\hline $\begin{array}{l}0 \\
\dot{1} \\
x \\
0 \\
\dot{0}\end{array}$ & 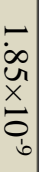 & 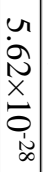 & 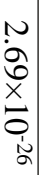 & $\mid \begin{array}{c}\omega \\
\infty \\
\dot{1} \\
\underline{x} \\
0 \\
\dot{v} \\
\dot{\sim}\end{array}$ & $\begin{array}{l}\omega \\
\underline{\Delta} \\
\underline{x} \\
\underline{0} \\
\underline{1}\end{array}$ & $\mid \begin{array}{l}u \\
\dot{\alpha} \\
\infty \\
x \\
0 \\
\dot{\omega} \\
\dot{d}\end{array}$ & 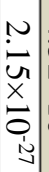 & 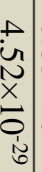 & 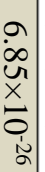 & 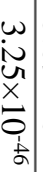 & $\begin{array}{c}r \\
\infty \\
0 \\
x \\
x \\
0 \\
b\end{array}$ & 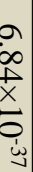 & $\begin{array}{l}\vec{t} \\
\dot{\omega} \\
\hat{x} \\
0 \\
0 \\
\dot{\omega} \\
\alpha\end{array}$ & $\begin{array}{c}\frac{1}{2} \\
\varrho \\
0 \\
0\end{array}$ \\
\hline $\begin{array}{l}u_{1} \\
u \\
\vec{u} \\
\times \\
\overline{0} \\
\frac{1}{\infty} \\
\end{array}$ & 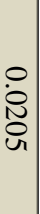 & $\left|\begin{array}{l}+ \\
\hat{N} \\
\pm \\
0 \\
x \\
0 \\
0 \\
ن \\
ن\end{array}\right|$ & $\begin{array}{l}\bar{\sigma} \\
\dot{\partial} \\
\infty \\
u \\
\times \\
\tilde{0} \\
b\end{array}$ & 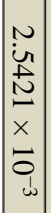 & $\begin{array}{l} \pm \\
\dot{y} \\
\text { a }\end{array}$ & 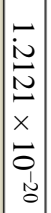 & $\begin{array}{l}n \\
8 \\
8 \\
0 \\
\times \\
\times \\
0 \\
\vdots \\
u\end{array}$ & 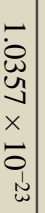 & $\begin{array}{c}\vec{\sim} \\
\vec{\omega} \\
\overrightarrow{+} \\
\times \\
\overrightarrow{0} \\
\infty\end{array}$ & 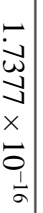 & $\begin{array}{l}0 \\
\text { in } \\
\dot{N} \\
\text { Na }\end{array}$ & 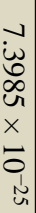 & 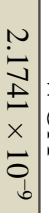 & $\&$ \\
\hline $\begin{array}{l}\infty \\
0 \\
\times \\
0 \\
\vdots \\
\dot{1}\end{array}$ & $\begin{array}{l}u \\
u \\
\vec{x} \\
\underline{x} \\
\underline{0} \\
\underline{i}\end{array}$ & 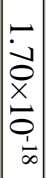 & 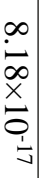 & 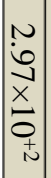 & 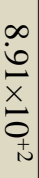 & $\mid \begin{array}{l}\bar{\sigma} \\
\overline{0} \\
x \\
0 \\
\dot{1}\end{array}$ & $\begin{array}{l}\infty \\
\dot{w} \\
\omega \\
x \\
0 \\
\dot{L}\end{array}$ & $\begin{array}{l}a \\
\vec{a} \\
x \\
0 \\
b\end{array}$ & $\begin{array}{l}0 \\
\dot{0} \\
x \\
\dot{x} \\
\dot{0}\end{array}$ & 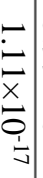 & $\begin{array}{l}u \\
0 \\
0 \\
x \\
x \\
0 \\
\dot{y} \\
0\end{array}$ & $\begin{array}{l}\infty \\
\overrightarrow{\vec{x}} \\
\frac{\vec{x}}{\sigma} \\
\dot{b}_{0}\end{array}$ & 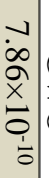 & $C$ \\
\hline $\begin{array}{l}\vec{v} \\
\dot{\phi} \\
\dot{\omega} \\
x \\
0 \\
\dot{\omega} \\
\dot{\omega}\end{array}$ & 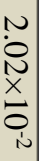 & \begin{tabular}{l}
0 \\
$\dot{0}$ \\
$\infty$ \\
$x$ \\
\hdashline \\
0 \\
$\dot{N}$ \\
\end{tabular} & $\begin{array}{l}w \\
\dot{u} \\
\overrightarrow{\underline{x}} \\
\vec{\sigma} \\
\dot{1}\end{array}$ & 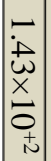 & 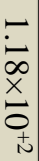 & 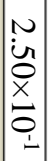 & $\begin{array}{c}\infty \\
\infty \\
\infty \\
\dot{x} \\
\underline{0} \\
0 \\
\underline{1}\end{array}$ & $\begin{array}{l}\infty \\
0 \\
-1\end{array}$ & $\begin{array}{l}\dot{+} \\
\overrightarrow{\tilde{x}} \\
\vec{\sigma} \\
\dot{ \pm}\end{array}$ & 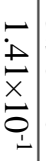 & $\begin{array}{l}\omega \\
\vdots \\
a \\
x \\
0 \\
0 \\
1\end{array}$ & 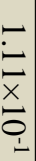 & $\begin{array}{l}N \\
\infty \\
\underset{x}{x} \\
0 \\
\dot{\nu}\end{array}$ & \\
\hline 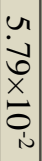 & $\begin{array}{l}\omega \\
\infty \\
\infty \\
x \\
x \\
0 \\
\dot{\sim}\end{array}$ & 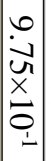 & 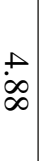 & $\begin{array}{l}\mathbf{c} \\
\infty \\
x \\
x \\
0 \\
+ \\
\omega\end{array}$ & 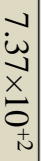 & $\begin{array}{l}\infty \\
\stackrel{N}{ }\end{array}$ & $\begin{array}{l}-\dot{\infty} \\
\dot{x} \\
x \\
0 \\
\dot{0} \\
\dot{ \pm}\end{array}$ & $\begin{array}{l}\omega \\
\infty \\
0 \\
x \\
x \\
0 \\
+ \\
\omega\end{array}$ & 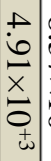 & $\begin{array}{c}\infty \\
\dot{u} \\
\vec{x} \\
x \\
0 \\
\dot{u} \\
\dot{u}\end{array}$ & 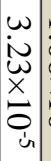 & 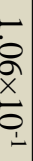 & $\left|\begin{array}{c}\omega \\
u \\
u \\
u \\
x \\
0 \\
i \\
i \\
\sim\end{array}\right|$ & $\exists$ \\
\hline 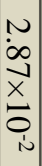 & $\begin{array}{l}\hat{a} \\
0 \\
\tilde{N} \\
x \\
0 \\
\dot{N}\end{array}$ & $\begin{array}{c}\underset{\dot{D}}{\infty} \\
\infty \\
\underset{\infty}{0} \\
\underset{\infty}{\infty}\end{array}$ & $\begin{array}{l}0 \\
\dot{u} \\
\underset{\tilde{x}}{x} \\
0 \\
\dot{b}\end{array}$ & $\begin{array}{l}w \\
\dot{o} \\
0 \\
x \\
x \\
0 \\
\pm \\
\pm\end{array}$ & 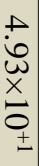 & 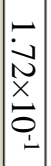 & 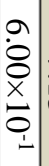 & $\stackrel{v}{\sim}$ & $\begin{array}{l}-\vec{x} \\
\dot{t} \\
\dot{x} \\
\overrightarrow{0} \\
\dot{ \pm}\end{array}$ & $\begin{array}{l}\dot{\infty} \\
\dot{+} \\
\dot{x} \\
\dot{\omega} \\
\dot{\omega}\end{array}$ & $\begin{array}{l}\sim \\
\tilde{D} \\
\dot{x} \\
0 \\
0 \\
+\end{array}$ & 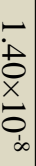 & $\begin{array}{l}\vec{b} \\
\dot{0} \\
\infty \\
x \\
0 \\
0 \\
b\end{array}$ & \\
\hline $\begin{array}{l}\omega \\
\tilde{N} \\
x \\
x \\
0 \\
\dot{\omega}\end{array}$ & $\begin{array}{l}a \\
\dot{D} \\
0 \\
x \\
0 \\
\dot{t} \\
+\end{array}$ & \begin{tabular}{l}
0 \\
$\dot{0}$ \\
$\infty$ \\
$x$ \\
\hdashline \\
0 \\
$i$ \\
\end{tabular} & $\begin{array}{c}w \\
\dot{\vec{u}} \\
\underline{x} \\
0 \\
\dot{1}\end{array}$ & 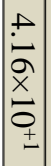 & $\begin{array}{l}u \\
\dot{u} \\
\dot{x} \\
x\end{array}$ & 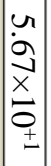 & 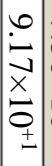 & $\begin{array}{l}v \\
\dot{w} \\
\hat{x} \\
\overrightarrow{0} \\
\dot{b}\end{array}$ & $\begin{array}{l}v \\
\vdots \\
0 \\
x \\
0 \\
\vdots \\
0\end{array}$ & $\begin{array}{l}u \\
\vec{x} \\
\underline{x}\end{array}$ & $\begin{array}{l}a \\
\dot{w} \\
\omega \\
x \\
0 \\
0 \\
\infty\end{array}$ & 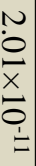 & 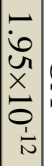 & $7^{1}$ \\
\hline
\end{tabular}

performance of eight other algorithms including Flow Direction Algorithm (FDA), Hide Object Game Optimizer (HOGO), Whale Optimization
Table 2. Results of RSLBO and other algorithms for high-dimensional Multimodal test functions

\begin{tabular}{|c|c|c|c|c|c|c|c|c|c|c|c|c|}
\hline & & I & & $T$ & & $\frac{T}{0}$ & & ] & & & & \\
\hline & 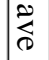 & $\frac{\tilde{a}}{2}$ & 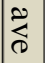 & $\stackrel{2}{\vec{a}}$ & 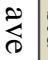 & $\frac{n}{2}$ & શ્ه & $\stackrel{\tilde{a}}{\overrightarrow{2}}$ & 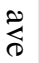 & $\stackrel{\mathscr{a}}{\tilde{L}}$ & עy & \\
\hline & $\stackrel{\circ}{\circ}$ & 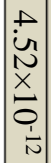 & 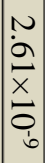 & 0 & 0 & 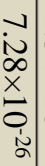 & 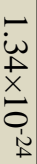 & 0 & 0 & $\begin{array}{l}\dot{\tilde{N}} \\
\frac{\bar{x}}{\sigma} \\
\frac{1}{u} \\
\frac{1}{u}\end{array}$ & 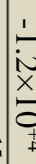 & $\begin{array}{l}\boldsymbol{\sigma} \\
\tilde{\omega} \\
\tilde{\sigma} \\
0 \\
0\end{array}$ \\
\hline & $\begin{array}{l}0 \\
\stackrel{0}{0} \\
\stackrel{0}{0}\end{array}$ & 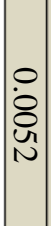 & 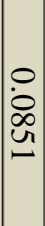 & 0 & 0 & $\begin{array}{l}a \\
\vec{w} \\
\vec{u} \\
\dot{x} \\
\vec{\sigma} \\
\frac{1}{N}\end{array}$ & $\begin{array}{l}0 \\
\text { aे } \\
0 \\
0 \\
x \\
x \\
0 \\
\frac{1}{N}\end{array}$ & $\begin{array}{l}N \\
\alpha \\
\omega \\
\\
\dot{A}\end{array}$ & $\frac{\vec{D}}{\stackrel{\vec{N}}{N}}$ & 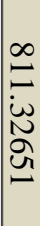 & 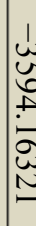 & \\
\hline 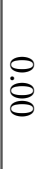 & $\stackrel{0}{8}$ & $\mid \begin{array}{l}w \\
\dot{0} \\
\alpha \\
x \\
0 \\
\dot{t} \\
+\end{array}$ & $\begin{array}{l}+ \\
\dot{\infty} \\
\dot{1} \\
x \\
\underline{0} \\
\dot{0} \\
\dot{u}\end{array}$ & 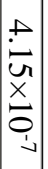 & $\begin{array}{l}-\vec{c} \\
\dot{y} \\
x \\
0 \\
\vdots \\
\vdots \\
0\end{array}$ & 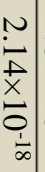 & 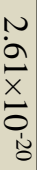 & 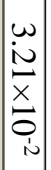 & 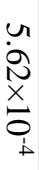 & $\begin{array}{l}\infty \\
\stackrel{\infty}{N} \\
\frac{x}{\alpha} \\
\frac{1}{n} \\
\frac{1}{N}\end{array}$ & $\begin{array}{l}\frac{1}{\tilde{x}} \\
\dot{x} \\
0 \\
1\end{array}$ & $t$ \\
\hline 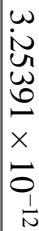 & 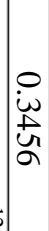 & $\mid \begin{array}{l}\infty \\
w \\
\pm \\
\pm \\
x \\
0 \\
0 \\
\frac{1}{N}\end{array}$ & 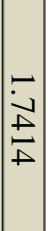 & $\mid$\begin{tabular}{l}
$\infty$ \\
0 \\
0 \\
0 \\
0 \\
\hdashline \\
$x$ \\
0 \\
$\vdots$ \\
$\frac{1}{v}$
\end{tabular} & : & $\begin{array}{l}\overrightarrow{0} \\
\infty \\
8 \\
0 \\
x \\
\overrightarrow{0} \\
\frac{1}{a}\end{array}$ & 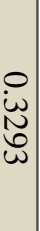 & $\mid \begin{array}{l}0 \\
i \\
0 \\
0 \\
N \\
x \\
0 \\
0 \\
\frac{1}{u} \\
\end{array}$ & $\begin{array}{l}\stackrel{+}{\tilde{O}} \\
\stackrel{\theta}{=}\end{array}$ & 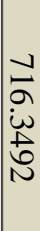 & $\begin{array}{l}1 \\
2 \\
2 \\
0 \\
\infty \\
\infty\end{array}$ & \\
\hline 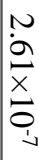 & $\begin{array}{l}0 \\
\dot{\delta} \\
\dot{+} \\
\underline{x} \\
\dot{0} \\
\dot{\alpha}\end{array}$ & $\begin{array}{c}a \\
\dot{N} \\
\tilde{\omega} \\
x \\
0 \\
\dot{b} \\
+\end{array}$ & $\begin{array}{l}-\bar{\omega} \\
\dot{\vec{x}} \\
\underline{x} \\
\underline{0} \\
\dot{u}\end{array}$ & $\begin{array}{l}\dot{\omega} \\
\dot{\vec{x}} \\
\vec{x} \\
\dot{\phi} \\
+\end{array}$ & 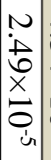 & $\begin{array}{l}\vec{t} \\
\dot{\omega} \\
\dot{x} \\
\underline{u} \\
\dot{\omega}\end{array}$ & 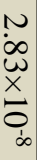 & 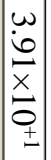 & $\begin{array}{l}\stackrel{p}{\infty} \\
\dot{\infty} \\
\underset{x}{x} \\
\underset{i}{0}\end{array}$ & $\begin{array}{l}\omega \\
\dot{b} \\
\dot{x} \\
\underline{\sigma} \\
\dot{ \pm}\end{array}$ & $\begin{array}{l}+ \\
\dot{x} \\
x \\
0 \\
\end{array}$ & 8 \\
\hline 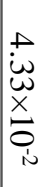 & 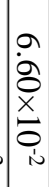 & 穴 & $\overrightarrow{\mathrm{N}}$ & 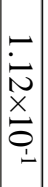 & $\begin{array}{l}u \\
\dot{\sim} \\
\overrightarrow{+} \\
\vec{x} \\
\dot{\partial} \\
\dot{1}\end{array}$ & 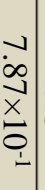 & $\dot{\vec{u}}$ & $\left|\begin{array}{c}\dot{\infty} \\
0 \\
x \\
x \\
0 \\
\pm \\
\pm\end{array}\right|$ & 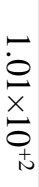 & $\begin{array}{l}0 \\
\dot{0} \\
x \\
x \\
0 \\
x \\
0 \\
0\end{array}$ & 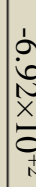 & \\
\hline 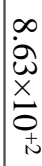 & 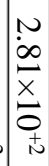 & 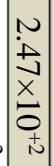 & 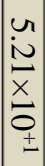 & $\begin{array}{l}N \\
\infty \\
0 \\
\dot{x} \\
x \\
0 \\
\dot{1}\end{array}$ & $\left|\begin{array}{c}\omega \\
0 \\
\underline{x} \\
0 \\
\dot{-}\end{array}\right|$ & $\stackrel{\infty}{=}$ & 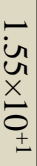 & $\begin{array}{c}\omega \\
\tilde{N} \\
\hat{x} \\
x \\
0 \\
\pm \\
\pm\end{array}$ & $\begin{array}{l}\stackrel{N}{N} \\
\underset{\sim}{x} \\
0 \\
0 \\
\pm\end{array}$ & $\begin{array}{l}\stackrel{N}{ } \\
\infty \\
\omega \\
x \\
\sigma \\
\dot{ \pm}\end{array}$ & $\underset{\alpha}{\infty} \underset{x}{x}$ & 寻 \\
\hline $\begin{array}{l}\dot{\theta} \\
\dot{\omega} \\
\underline{x} \\
x \\
\dot{0} \\
\dot{i}\end{array}$ & $\begin{array}{l}\hat{a} \\
\hat{\alpha} \\
\hat{x} \\
0 \\
0 \\
\dot{v}\end{array}$ & 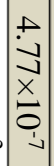 & $\begin{array}{l}\infty \\
\dot{b} \\
w \\
x \\
\dot{x} \\
\dot{\infty} \\
\dot{\infty}\end{array}$ & 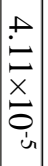 & 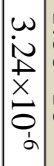 & $\begin{array}{l}\dot{0} \\
\infty \\
x \\
\dot{0} \\
\frac{1}{\sigma}\end{array}$ & 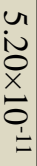 & $\mid \begin{array}{c}+ \\
\dot{0} \\
\underline{x} \\
\underline{0} \\
\pm \\
\pm\end{array}$ & $\begin{array}{l}\overline{\dot{v}} \\
\underset{\dot{x}}{x} \\
\stackrel{0}{0}\end{array}$ & $\begin{array}{l}\vec{x} \\
\dot{N} \\
\dot{x} \\
\underline{\sigma} \\
\dot{0}\end{array}$ & 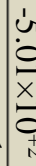 & 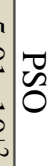 \\
\hline $\begin{array}{l}\mathbf{x} \\
\underset{0}{0} \\
\mathbf{i} \\
\end{array}$ & 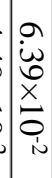 & 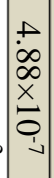 & $\begin{array}{l}0 \\
\dot{\hat{a}} \\
\underline{x} \\
\hat{0} \\
\infty \\
\infty\end{array}$ & 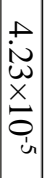 & 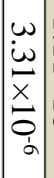 & $\underline{\bar{x}}$ & 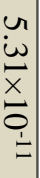 & $\mid \begin{array}{l}+ \\
\overrightarrow{\bar{x}} \\
\stackrel{x}{\circ} \\
\pm\end{array}$ & $\mid \begin{array}{l}\dot{N} \\
\omega \\
x \\
0 \\
0 \\
-1\end{array}$ & 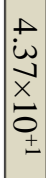 & $\underset{\square}{x}$ & 1 \\
\hline
\end{tabular}

Algorithm (WOA), Grey Wolf Optimization (GWO), Teaching-Learning-Based Optimization (TLBO), Gravitational Search Algorithm (GSA), Particle 
Table 3. Results of RSLBO and other algorithms for fixed-dimensional Multimodal test functions

\begin{tabular}{|c|c|c|c|c|c|c|c|c|c|c|c|c|c|c|c|c|c|c|c|c|}
\hline \multicolumn{2}{|c|}{ 恿 } & & \multicolumn{2}{|c|}{$\mathbb{N}^{\mathbb{N}}$} & \multicolumn{2}{|c|}{${ }_{0}^{T}$} & \multicolumn{2}{|c|}{$T$} & \multicolumn{2}{|c|}{$T$} & \multicolumn{2}{|c|}{ I } & \multicolumn{2}{|c|}{ I } & \multicolumn{2}{|l|}{$T$} & & \\
\hline$\frac{2}{2}$ & 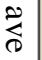 & $\stackrel{\mathscr{a}}{2}$ & $\stackrel{\overbrace{}}{\circlearrowright}$ & $\frac{n}{2}$ & $\underset{ه}{\stackrel{\infty}{\alpha}}$ & $\stackrel{n}{2}$ & 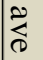 & $\frac{n}{2}$ & 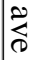 & $\frac{n}{2}$ & 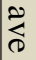 & $\frac{\mathscr{a}}{2}$ & 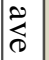 & $\stackrel{n}{2}$ & 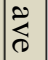 & $\stackrel{n}{2}$ & 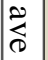 & $\frac{n}{2}$ & 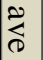 & \\
\hline 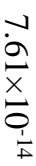 & 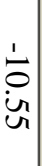 & $\begin{array}{c}0 \\
\dot{N} \\
\infty \\
x \\
0 \\
0 \\
\frac{1}{t}\end{array}$ & $\begin{array}{l}1 \\
0 \\
+ \\
0\end{array}$ & 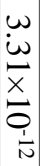 & $\frac{1}{0}$ & 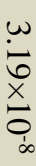 & $\begin{array}{l}\omega \\
\dot{\omega}\end{array}$ & $\begin{array}{l}\dot{0} \\
\dot{\infty} \\
x \\
0 \\
\vdots \\
\vdots \\
\sigma\end{array}$ & 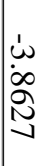 & $\begin{array}{l}+\overrightarrow{0} \\
\dot{\infty} \\
x \\
x \\
0 \\
0 \\
\dot{\omega}\end{array}$ & $\omega$ & 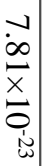 & 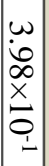 & $\begin{array}{l}0 \\
0 \\
\overrightarrow{0} \\
x \\
0 \\
\dot{1} \\
\dot{v}\end{array}$ & $\begin{array}{l}\frac{1}{0} \\
\frac{\omega}{a} \\
\frac{0}{a}\end{array}$ & 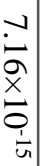 & $\left|\begin{array}{l}a \\
\dot{A} \\
\hat{N} \\
x \\
0 \\
\vdots \\
\alpha\end{array}\right|$ & $\begin{array}{l}\underbrace{u} \\
\frac{\vec{x}}{0} \\
\frac{1}{\infty}\end{array}$ & $\begin{array}{l}0 \\
6 \\
0 \\
\infty\end{array}$ & \\
\hline $\begin{array}{l}\infty \\
\infty \\
\stackrel{0}{x} \\
0 \\
0\end{array}$ & $\begin{array}{l}\frac{1}{0} \\
\dot{i} \\
\dot{\omega} \\
\end{array}$ & $\begin{array}{c}N \\
\infty \\
0 \\
u r \\
+ \\
\times \\
\overrightarrow{0} \\
0 \\
= \\
=\end{array}$ & $\begin{array}{l}\frac{1}{0} \\
0 \\
10 \\
0\end{array}$ & 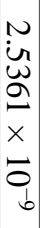 & $\frac{1}{0}$ & 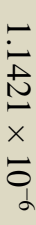 & & $\begin{array}{l}\overrightarrow{+} \\
\stackrel{N}{+} \\
\stackrel{N}{\infty} \\
\infty \\
x \\
\overrightarrow{0} \\
\frac{1}{+}\end{array}$ & $\begin{array}{l}\omega \\
\infty \\
\alpha\end{array}$ & $\begin{array}{l}-\dot{0} \\
0 \\
\infty \\
\infty \\
+ \\
x \\
0 \\
0 \\
\frac{1}{n}\end{array}$ & $\omega$ & $\begin{array}{l}0 \\
\dot{n} \\
w \\
u \\
x \\
\overline{0} \\
\frac{1}{v}\end{array}$ & $\begin{array}{l}\omega \\
\dot{0} \\
0 \\
x \\
x \\
0 \\
\dot{1} \\
\end{array}$ & $\begin{array}{l}+\vec{c} \\
\vec{a} \\
\vec{\omega} \\
\vec{u} \\
\times \\
\overrightarrow{0} \\
0 \\
\frac{1}{f}\end{array}$ & $\frac{1}{\dot{\omega}}$ & 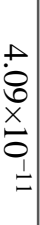 & $\begin{array}{l}\circ \\
\stackrel{0}{\odot} \\
\stackrel{\omega}{\omega}\end{array}$ & 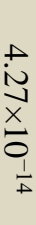 & $\begin{array}{l}0 \\
0 \\
0 \\
\infty \\
\infty\end{array}$ & \\
\hline 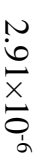 & $\begin{array}{l}1 \\
0 \\
\text { ú } \\
\text { ú }\end{array}$ & 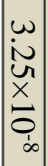 & $\begin{array}{l}\overrightarrow{0} \\
+ \\
0\end{array}$ & 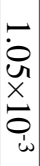 & $\frac{1}{0}$ & 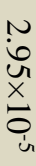 & & 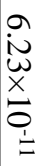 & & $\begin{array}{l}\dot{a} \\
\frac{\hat{x}}{\sigma} \\
\frac{1}{6}\end{array}$ & $\omega$ & 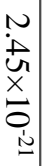 & $\begin{array}{c}\omega \\
\dot{0} \\
\infty \\
x \\
0 \\
\dot{0}\end{array}$ & $\begin{array}{l}w \\
\vdots \\
\tilde{a} \\
\underline{x} \\
0 \\
\vdots \\
\frac{1}{0}\end{array}$ & $\frac{1}{\dot{\omega}}$ & 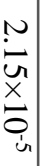 & 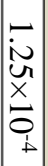 & 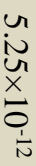 & $\begin{array}{l}0 \\
0 \\
\overline{0} \\
x \\
0 \\
\underline{0}\end{array}$ & \\
\hline 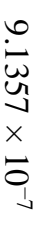 & $\begin{array}{l}1 \\
0 \\
8 \\
8 \\
0 \\
\omega\end{array}$ & 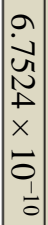 & $\begin{array}{l}\infty \\
\infty \\
\underline{0}\end{array}$ & $\begin{array}{l}N \\
w \\
\infty \\
\sigma \\
0 \\
x \\
0 \\
1 \\
v\end{array}$ & $\frac{1}{4}$ & 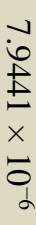 & $\begin{array}{l}\omega \\
\dot{\sim} \\
\perp\end{array}$ & 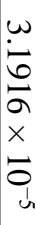 & $\underset{\infty}{\omega}$ & $\begin{array}{l}y \\
0 \\
0 \\
\infty \\
+ \\
x \\
x \\
0 \\
0 \\
u\end{array}$ & $\omega$ & 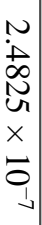 & 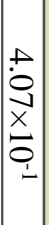 & $\begin{array}{l}0 \\
0 \\
\omega \\
0 \\
0 \\
\times \\
\sigma \\
\alpha\end{array}$ & $\frac{1}{\dot{\omega}}$ & $\begin{array}{l}w \\
\dot{0} \\
\dot{b} \\
0 \\
x \\
0 \\
0 \\
\infty \\
\infty\end{array}$ & $\mid \begin{array}{l}0 \\
\dot{8} \\
\dot{1} \\
0\end{array}$ & 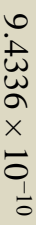 & $\begin{array}{l}0 \\
0 \\
0 \\
\infty \\
\infty\end{array}$ & \\
\hline - & $\begin{array}{l}\dot{1} \\
\dot{+} \\
\dot{b}\end{array}$ & & $\begin{array}{l}\dot{\omega} \\
\dot{\nabla}\end{array}$ & $\dot{\infty}$ & 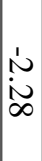 & $\frac{\overrightarrow{\bar{x}}}{\underline{x}}$ & 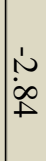 & 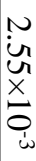 & ư & 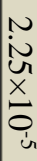 & w & $\begin{array}{l}\dot{v} \\
\dot{\vec{x}} \\
\dot{0} \\
\dot{t}\end{array}$ & $\begin{array}{c}w \\
\dot{0} \\
\infty \\
x \\
0 \\
0 \\
\underline{-}\end{array}$ & 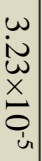 & $\frac{1}{\dot{2}}$ & $\begin{array}{l}\omega \\
\dot{w} \\
\underline{x} \\
x \\
\vdots \\
\dot{\omega}\end{array}$ & 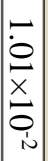 & $\begin{array}{l}a \\
\infty \\
\hat{\alpha} \\
\underline{x} \\
\underline{0}\end{array}$ & i & \\
\hline & $\begin{array}{l}\dot{\infty} \\
\doteq \\
\doteq\end{array}$ & $\begin{array}{l}\omega \\
\dot{N}\end{array}$ & $\begin{array}{l}\dot{\infty} \\
\dot{y} \\
0\end{array}$ & $\underline{\sigma}$ & $\frac{1}{i \omega}$ & 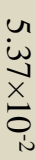 & $\underset{\omega}{\omega}$ & 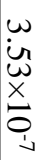 & نّ & 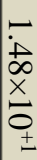 & ر & 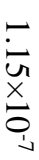 & $\begin{array}{c}\omega \\
i \\
\infty \\
x \\
x \\
0 \\
\vdots \\
i\end{array}$ & $\begin{array}{l}\stackrel{+}{\vec{v}} \\
\stackrel{\vec{x}}{x} \\
\overrightarrow{0} \\
\dot{\infty}\end{array}$ & $\frac{1}{\text { Dิ }}$ & $\begin{array}{l}- \\
\tilde{a} \\
\hat{x} \\
\vdots \\
\vdots \\
1\end{array}$ & 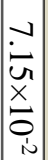 & $\begin{array}{l}\stackrel{0}{\vec{t}} \\
\stackrel{\vec{x}}{0} \\
\underline{\underline{0}}\end{array}$ & $\begin{array}{l}0 \\
0 \\
\infty \\
x \\
0 \\
0 \\
\pm\end{array}$ & \\
\hline & $\frac{n}{a}$ & $\left|\begin{array}{c}N \\
0 \\
0 \\
x \\
x \\
0 \\
\vdots \\
\vdots\end{array}\right|$ & $\frac{1}{8}$ & $\overline{\tilde{\sigma}}$ & سָ & $\begin{array}{l}\stackrel{p}{\dot{x}} \\
\underset{0}{0}\end{array}$ & $\stackrel{\stackrel{N}{*}}{\underline{*}}$ & $\begin{array}{l}0 \\
\dot{a} \\
\dot{b} \\
x \\
0 \\
\dot{b}\end{array}$ & سَّ & 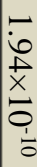 & ر & 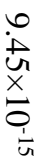 & $\begin{array}{l}\omega \\
\dot{0} \\
\infty \\
x \\
0 \\
\dot{1} \\
\dot{1}\end{array}$ & $\begin{array}{l}\omega \\
\dot{\alpha} \\
\dot{x} \\
\dot{x} \\
\dot{\infty}\end{array}$ & $\frac{1}{0}$ & $\begin{array}{l}\omega \\
\dot{\Delta} \\
x \\
\dot{\omega} \\
\dot{\omega}\end{array}$ & 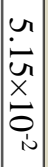 & $\bar{N}$ & 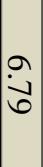 & 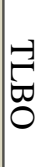 \\
\hline in & $\frac{6}{6}$ & $\begin{array}{l}\omega \\
\dot{\infty} \\
\infty\end{array}$ & $\begin{array}{l}\dot{1} \\
\dot{u} \\
u\end{array}$ & $\stackrel{N}{N}$ & ' & $\frac{x}{x}$ & رِ & 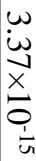 & سـ & $\begin{array}{l}\text { U } \\
x \\
x \\
0\end{array}$ & 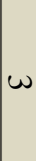 & $\begin{array}{l}0 \\
\stackrel{0}{\omega} \\
\dot{x} \\
x \\
0 \\
\vdots \\
\sigma\end{array}$ & $\begin{array}{c}w \\
\dot{0} \\
\infty \\
x \\
0 \\
0 \\
\dot{1}\end{array}$ & $\stackrel{\circ}{\circ}$ & $\frac{1}{\mathfrak{O}}$ & $\begin{array}{l}N \\
w \\
\infty \\
x \\
\tilde{D} \\
\dot{\omega}\end{array}$ & $\mid \begin{array}{c}0 \\
0 \\
0 \\
x \\
x \\
0 \\
\dot{\omega} \\
\end{array}$ & $\underset{N}{N}$ & 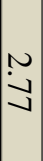 & 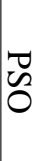 \\
\hline שِ & & 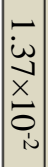 & o & 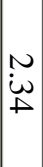 & ת & $\begin{array}{l}\underline{w} \\
\underset{x}{x} \\
\underline{0}\end{array}$ & رِ & 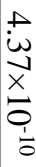 & ' & $\begin{array}{l}w_{\omega} \\
x \\
\underline{0}\end{array}$ & ر & 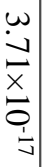 & $\begin{array}{l}w \\
\dot{0} \\
\infty \\
x \\
\underline{0} \\
\dot{1}\end{array}$ & $\begin{array}{l}\dot{+} \\
\bar{\sigma} \\
x \\
0 \\
\dot{0}\end{array}$ & $\frac{1}{0}$ & $\begin{array}{l}N \\
\dot{\omega} \\
0 \\
x \\
0 \\
\dot{\omega} \\
\dot{\omega}\end{array}$ & $\begin{array}{l}v \\
\dot{w} \\
\hat{x} \\
x \\
0 \\
\dot{v} \\
\dot{v}\end{array}$ & 辛 & $\vec{i}$ & 2 \\
\hline
\end{tabular}

Swarm Optimization (PSO), and Genetic Algorithm (GA). The simulation results showed that the proposed RSLBO has a high capability in solving optimization problems. Also, the analysis of the performance of the RSLBO in comparison with the eight mentioned algorithms showed the superiority of the proposed RSLBO in providing more effective and competitive solutions. 
The authors offer several ideas and suggestions for future studies. The design of the binary version as well as the multi-objective version of the proposed RSLBO are the special perspectives of this paper. On the other hand, the use of the RSLBO in solving various optimization problems in different fields of science and real-life problems is another potential of the proposed algorithm for future research.

\section{Conflicts of Interest}

The authors declare no conflict of interest.

\section{Author Contributions}

Conceptualization, F.A.Z.; methodology, M.D.; software, M.D.; validation, F.A.Z., O.P.M., and M.D.; formal analysis, O.P.M. and F.A.Z.; investigation, M.D., F.A.Z., and O.P.M.; resources, M.D.; data curation, F.A.Z.; writing - original draft preparation, M.D.; writing - review and editing, O.P.M. and F.A.Z.; visualization, M.D; supervision, M.D.; project administration, F.A.Z.; funding acquisition, O.P.M.

\section{References}

[1] M. Dehghani, Z. Montazeri, O. P. Malik, G. Dhiman, and V. Kumar, "BOSA: Binary orientation search algorithm", Int. J. Innov. Technol. Explor. Eng.(IJITEE), Vol. 9, pp. 5306-5310, 2019.

[2] M. Dehghani, Z. Montazeri, A. Dehghani, R. A. R. Mendoza, H. Samet, J. M. Guerrero, and G. Dhiman, "MLO: Multi leader optimizer", International Journal of Intelligent Engineering and Systems, Vol. 13, pp. 364-373, 2020.

[3] M. Dehghani, Z. Montazeri, A. Dehghani, and O. P. Malik, "GO: Group optimization", Gazi University Journal of Science, Vol. 33, No. 2, pp. 381-392, 2020.

[4] G. Dhiman, K. K. Singh, M. Soni, A. Nagar, M. Dehghani, A. Slowik, A. Kaur, A. Sharma, E. H. Houssein, and K. Cengiz, "MOSOA: A new multi-objective seagull optimization algorithm", Expert Systems with Applications, pp. 114150, 2020.

[5] G. Dhiman, M. Garg, A. Nagar, V. Kumar, and M. Dehghani, "A novel algorithm for global optimization: Rat swarm optimizer", Journal of Ambient Intelligence and Humanized Computing, pp. 1-26, 2020.

[6] M. Dehghani, Z. Montazeri, A. Dehghani, H. Samet, C. Sotelo, D. Sotelo, A. Ehsanifar, O. P. Malik, J. M. Guerrero, and G. Dhiman, "DM: Dehghani Method for modifying optimization algorithms", Applied Sciences, Vol. 10, No. 21, pp. 7683, 2020.

[7] M. Dehghani, Z. Montazeri, G. Dhiman, O. Malik, R. M. Menendez, R. A. R. Mendoza, A. Dehghani, J. M. Guerrero, and L. P. Arroyo, "A spring search algorithm applied to engineering optimization problems", Applied Sciences, Vol. 10, No. 18, pp. 6173, 2020.

[8] M. Dehghani, Z. Montazeri, and O. P. Malik, "ENERGY COMMITMENT: A PLANNING OF ENERGY CARRIER BASED ON ENERGY CONSUMPTION", Electrical Engineering \& Electromechanics, No. 4, pp. 6972, 2019.

[9] M. Dehghani, M. Mardaneh, O. P. Malik, J. M. Guerrero, C. Sotelo, D. Sotelo, M. N. Heris, K. A. Haddad, and R. A. R. Mendoza, "Genetic Algorithm for Energy Commitment in a Power System Supplied by Multiple Energy Carriers", Sustainability, Vol. 12, No. 23, p. 10053, 2020.

[10] M. Dehghani, M. Mardaneh, O. P. Malik, J. M. Guerrero, R. M. Menendez, R. A. R. Mendoza, J. Matas, and A. Abusorrah, "Energy Commitment for a Power System Supplied by Multiple Energy Carriers System using Following Optimization Algorithm", Applied Sciences, Vol. 10, No. 17, pp. 5862, 2020.

[11] H. Rezk, A. Fathy, M. Aly, and M. N. F. Ibrahim, "Energy management control strategy for renewable energy system based on spotted hyena optimizer", Computers, Materials \& Continua, Vol. 67, No. 2, pp. 2271-2281, 2021.

[12] A. Ehsanifar, M. Dehghani, and M. Allahbakhshi, "Calculating The Leakage Inductance for Transformer Inter-Turn Fault Detection Using Finite Element Method", In: Proc. of Iranian Conference on Electrical Engineering (ICEE), pp.1372-1377, 2017.

[13] M. Dehghani, Z. Montazeri, and O. Malik, "Optimal sizing and placement of capacitor banks and distributed generation in distribution systems using spring search algorithm", International Journal of Emerging Electric Power Systems, Vol. 21, No. 1, 2020.

[14] M. Dehghani, Z. Montazeri, O. P. Malik, K. A. Haddad, J. M. Guerrero, and G. Dhiman, "A New Methodology Called Dice Game Optimizer for Capacitor Placement in Distribution Systems", Electrical Engineering \& Electromechanics, No. 1, pp. 61-64, 2020.

[15] S. Dehbozorgi, A. Ehsanifar, Z. Montazeri, M. Dehghani, and A. Seifi, "Line Loss Reduction and Voltage Profile Improvement in Radial Distribution Networks Using Battery Energy Storage System", In: Proc. of IEEE 4th 
International Conference on Knowledge-Based Engineering and Innovation (KBEI), pp. 02150219, 2017.

[16] Z. Montazeri and T. Niknam, "Optimal utilization of electrical energy from power plants based on final energy consumption using gravitational search algorithm", Electrical Engineering \& Electromechanics, No. 4, pp. 7073, 2018.

[17] M. Dehghani, M. Mardaneh, Z. Montazeri, A. Ehsanifar, M. J. Ebadi, and O. M. Grechko, "Spring search algorithm for simultaneous placement of distributed generation and capacitors", Electrical Engineering \& Electromechanics, No. 6, pp. 68-73, 2018.

[18] M. Premkumar, R. Sowmya, P. Jangir, K. S. Nisar, and M. Aldhaifallah, "A New Metaheuristic Optimization Algorithms for Brushless Direct Current Wheel Motor Design Problem", CMC-Computers Materials \& Continua, Vol. 67, No. 2, pp. 2227-2242, 2021.

[19] M. Dehghani, Z. Montazeri, A. Ehsanifar, A. R. Seifi, M. J. Ebadi, and O. M. Grechko, "Planning of energy carriers based on final energy consumption using dynamic programming and particle swarm optimization", Electrical Engineering \& Electromechanics, No. 5, pp. 6271, 2018.

[20] Z. Montazeri and T. Niknam, "Energy Carriers Management Based on Energy Consumption", In: Proc. of IEEE 4th International Conference on Knowledge-Based Engineering and Innovation (KBEI), pp. 0539-0543, 2017.

[21] J. Kennedy and R. Eberhart, "Particle swarm optimization", In: Proc. of ICNN'95International Conference on Neural Networks, Vol. 4, pp. 1942-1948, 1995.

[22] M. Dehghani, M. Mardaneh, J. M. Guerrero, O. P. Malik, R. A. R. Mendoza, J. Matas, J. C. Vasquez, and L. P. Arroyo, "A new "Doctor and Patient" optimization algorithm: An application to energy commitment problem", Applied Sciences, Vol. 10, No. 17, p. 5791, 2020.

[23] S. Mirjalili, S. M. Mirjalili, and A. Lewis, "Grey wolf optimizer", Advances in Engineering Software, Vol. 69, pp. 46-61, 2014.

[24] R. V. Rao, V. J. Savsani, and D. Vakharia, "Teaching-learning-based optimization: a novel method for constrained mechanical design optimization problems", Computer-Aided Design, Vol. 43, No. 3, pp. 303-315, 2011.

[25] S. Mirjalili and A. Lewis, "The whale optimization algorithm", Advances in Engineering Software, Vol. 95, pp. 51-67, 2016.
[26] S. A. Doumari, H. Givi, M. Dehghani, Z. Montazeri, V. Leiva, and J. M. Guerrero, "A New Two-Stage Algorithm for Solving Optimization Problems", Entropy, Vol. 23, No. 4, p. 491, 2021.

[27] M. Dehghani, Z. Montazeri, and Š. Hubálovský, "GMBO: Group Mean-Based Optimizer for Solving Various Optimization Problems", Mathematics, Vol. 9, No. 11, p. 1190, 2021.

[28] M. Dehghani, M. Mardaneh, O. P. Malik, and S. M. NouraeiPour, "DTO: Donkey Theorem Optimization", In: Proc. of 27th Iranian Conference on Electrical Engineering (ICEE), pp.1855-1859, 2019.

[29] A. Sadeghi, S. A. Doumari, M. Dehghani, Z. Montazeri, P. Trojovský, and H. J. Ashtiani, "A New "Good and Bad Groups-Based Optimizer" for Solving Various Optimization Problems", Applied Sciences, Vol. 11, No. 10, p. 4382, 2021.

[30] H. Givi, M. Dehghani, Z. Montazeri, R. M. Menendez, R. A. R. Mendoza, and N. Nouri, "GBUO: "The Good, the Bad, and the Ugly" Optimizer", Applied Sciences, Vol. 11, No. 5, p. 2042, 2021.

[31] M. Dehghani, M. Mardaneh, and O. Malik, "FOA:'Following'Optimization Algorithm for solving Power engineering optimization problems", Journal of Operation and Automation in Power Engineering, Vol. 8, No. 1, pp. 57-64, 2020.

[32] S. Kirkpatrick, C. D. Gelatt, and M. P. Vecchi, "Optimization by simulated annealing", Science, Vol. 220, No. 4598, pp. 671-680, 1983.

[33] E. Rashedi, H. N. Pour, and S. Saryazdi, "GSA: a gravitational search algorithm", Information Sciences, Vol. 179, No. 13, pp. 2232-2248, 2009.

[34] M. Dehghani, Z. Montazeri, A. Dehghani, and A. Seifi, "Spring Search Algorithm: A New MetaHeuristic Optimization Algorithm Inspired by Hooke's Law", In: Proc. of IEEE 4th International Conference on Knowledge-Based Engineering and Innovation (KBEI), pp. 02100214, 2017.

[35] H. Karami, M. V. Anaraki, S. Farzin, and S. Mirjalili, "Flow Direction Algorithm (FDA): A Novel Optimization Approach for Solving Optimization Problems", Computers \& Industrial Engineering, Vol. 156, pp. 107224, 2021.

[36] M. Dehghani and H. Samet, "Momentum search algorithm: A new meta-heuristic optimization algorithm inspired by momentum conservation law", SN Applied Sciences, Vol. 2, No. 10, pp. 1-15, 2020. 
[37] M. Dehghani, Z. Montazeri, A. Dehghani, N. Nouri, and A. Seifi, "BSSA: Binary Spring Search Algorithm", In: Proc. of IEEE 4th International Conference on Knowledge-Based Engineering and Innovation (KBEI), pp. 02200224, 2017.

[38] F. A. Hashim, E. H. Houssein, M. S. Mabrouk, W. A. Atabany, and S. Mirjalili, "Henry gas solubility optimization: A novel physics-based algorithm", Future Generation Computer Systems, Vol. 101, pp. 646-667, 2019.

[39] R. A. Formato, "Central force optimization", Prog Electromagn Res, Vol. 77, pp. 425-491, 2007.

[40] M. Dehghani, M. Mardaneh, J. M. Guerrero, O. Malik, and V. Kumar, "Football game based optimization: An application to solve energy commitment problem", International Journal of Intelligent Engineering and Systems, Vol. 13, pp. 514-523, 2020.

[41] M. Dehghani, Z. Montazeri, S. Saremi, A. Dehghani, O. P. Malik, K. A. Haddad, and J. M. Guerrero, "HOGO: Hide Objects Game Optimization", International Journal of Intelligent Engineering and Systems, Vol. 13, No. 4, pp. 216-225, 2020.

[42] M. Dehghani, Z. Montazeri, H. Givi, J. M. Guerrero, and G. Dhiman, "Darts game optimizer: A new optimization technique based on darts game", International Journal of Intelligent Engineering and Systems, Vol. 13, pp. 286-294, 2020.

[43] M. Dehghani, Z. Montazeri, O. P. Malik, A. Ehsanifar, and A. Dehghani, "OSA: Orientation search algorithm", International Journal of Industrial Electronics, Control and Optimization, Vol. 2, No. 2, pp. 99-112, 2019.

[44] S. A. Doumari, H. Givi, M. Dehghani, and O. P. Malik, "Ring Toss Game-Based Optimization Algorithm for Solving Various Optimization Problems", International Journal of Intelligent Engineering and Systems, Vol. 14, No. 3, pp. 545-554, 2021.

[45] M. Dehghani, Z. Montazeri, O. P. Malik, H. Givi, and J. M. Guerrero, "Shell game optimization: A novel game-based algorithm", International Journal of Intelligent Engineering and Systems, Vol. 13, No. 3, pp. 246-255, 2020.

[46] A. Bose, T. Biswas, and P. Kuila, "A novel genetic algorithm based scheduling for multicore systems", Smart Innovations in Communication and Computational Sciences, pp. 45-54, 2019.
[47] S. A. Hofmeyr and S. Forrest, "Architecture for an artificial immune system", EVolutionary Computation, Vol. 8, No. 4, pp. 443-473, 2000.

[48] L. J. Fogel, A. J. Owens, and M. J. Walsh, "Artificial intelligence through simulated eVolution", 1966.

[49] G. Huang, "Artificial infectious disease optimization: A SEIQR epidemic dynamic model-based function optimization algorithm", Swarm and EVolutionary Computation, Vol. 27, pp. 31-67, 2016.

[50] H. G. Beyer and H. P. Schwefel, "EVolution strategies-A comprehensive introduction", Natural Computing, Vol. 1, No. 1, pp. 3-52, 2002.

[51] J. R. Koza, "Genetic programming as a means for programming computers by natural selection", Statistics and Computing, Vol. 4, No. 2, pp. 87-112, 1994.

[52] R. G. Reynolds, "An introduction to cultural algorithms", In: Proc. of the third annual Conference on eVolutionary Programming, 1994, Vol. 24, pp. 131-139, 1994.

[53] S. Das and P. N. Suganthan, "Differential eVolution: a survey of the state-of-the-art", IEEE Transactions on EVolutionary Computation, Vol. 15, No. 1, pp. 4-31, 2011.

[54] D. Simon, "Biogeography-based optimization", IEEE Transactions on EVolutionary Computation, Vol. 12, No. 6, pp. 702-713, 2008. 